\title{
Brain activity patterns uniquely supporting visual feature integration after traumatic brain injury
}

\author{
Anjali Raja Beharelle ${ }^{1,2}$, Danielle Tisserand ${ }^{1,2}$, Donald T. Stuss ${ }^{1,2,3}$, Anthony R. Mclntosh ${ }^{1,2}$ and \\ Brian Levine ${ }^{1,2,3}$
}

${ }^{1}$ Rotman Research Institute of Baycrest Centre, University of Toronto, Toronto, ON, Canada

${ }^{2}$ Department of Psychology, University of Toronto, Toronto, ON, Canada

${ }^{3}$ Department of Medicine (Neurology), University of Toronto, Toronto, ON, Canada

\section{Edited by:}

Robert T. Knight, University of

California Berkeley, USA

Reviewed by:

Robert T. Knight, University of California Berkeley, USA

Tracy L. Luks, University of California

San Francisco, USA

\section{${ }^{*}$ Correspondence:}

Anjali Raja Beharelle, Rotman

Research Institute, Baycrest Centre for Geriatric Care, 3560 Bathurst

Street, Toronto, Canada ON M6A 2 E1. e-mail:anjali.raja@utoronto.ca
Traumatic brain injury (TBI) patients typically respond more slowly and with more variability than controls during tasks of attention requiring speeded reaction time. These behavioral changes are attributable, at least in part, to diffuse axonal injury (DAl), which affects integrated processing in distributed systems. Here we use a multivariate method sensitive to distributed neural activity to compare brain activity patterns of patients with chronic phase moderate to-severe TBI to those of controls during performance on a visual feature integration task assessing complex attentional processes that has previously shown sensitivity to TBI. The TBI patients were carefully screened to be free of large focal lesions that can affect performance and brain activation independently of DAI. The task required subjects to hold either one or three features of a Target in mind while suppressing responses to distracting information. In controls, the multi-feature condition activated a distributed network including limbic, prefrontal, and medial temporal structures. TBI patients engaged this same network in the single-feature and baseline conditions. In multi-feature presentations, TBI patients alone activated additional frontal, parietal, and occipital regions. These results are consistent with neuroimaging studies using tasks assessing different cognitive domains, where increased spread of brain activity changes was associated with TBI. Our results also extend previous findings that brain activity for relatively moderate task demands in TBI patients is similar to that associated with of high task demands in controls.

Keywords: traumatic brain injury, diffuse axonal injury, attention, feature integration, fMRI, partial least squares

\section{INTRODUCTION}

Impairments in attention, cognitive control, and speed of information processing have been frequently observed after traumatic brain injury (TBI; Stuss et al., 1989; Spikman et al., 1996; Mathias and Wheaton, 2007). Deficits in these processes, which are considered to involve large-scale neural interactions (Mesulam, 1998), may arise in part due to the diffuse underlying neuropathology present after TBI. While focal lesions play a significant role in functional outcome, the presence of diffuse axonal injury (DAI) is more ubiquitous after TBI (Gentleman et al., 1995), and profound cognitive deficits have been observed in patients with DAI in the absence of focal lesions (Scheid et al., 2006). In DAI, rapid deceleration of the head results in disruption of ionic homeostasis, cytoskeletal misalignment, swelling of the axonal segment, and ultimate deafferentation of the axon from its synaptic field (Povlishock and Christman, 1995; Maxwell et al., 1997), ultimately causing volume loss throughout the cerebrum, especially in patients with moderate-to-severe injury (Levine et al., 2008). Prefrontal cortical regions may be especially vulnerable due to their extensive reentrant connections with most cortical and subcortical regions (Petrides and Pandya, 1999). It has been suggested that functional recovery of the cognitive and behavioral sequelae of TBI may involve restoration of integration between frontal areas and modality-specific posterior regions (Chen et al., 2006).
Functional neuroimaging studies of attention and working memory have generally shown more widespread activation in TBI patients as compared to healthy subjects, particularly in frontal and temporal-parietal areas (Christodoulou et al., 2001; Levine et al., 2002; McAllister et al., 2006; Kim et al., 2008; Turner and Levine, 2008). This increase in the extent of neural activity after TBI is similar to patterns that have been observed during aging, where older participants show increased bilateral activity across a range of memory and attentional tasks in prefrontal cortex (Cabeza et al., 2002, 2004; de Chastelaine et al., 2011) as well as posterior parietal cortices (Huang et al., 2011; Vallesi et al., 2011) Greater recruitment of regions homologous to areas used for cognitive processes in controls has also been found (Christodoulou et al., 2001; Levine et al., 2002; Maruishi et al., 2007, for exception see Perlstein et al., 2004). Most previous studies have been confounded methodologically either by the fact that TBI patients with and without focal lesions were mixed within their samples or because differences in behavioral performance between TBI patients and controls made it difficult to disentangle brain activity underlying performance effects from neural changes resulting from head injury (Levine et al., 2006). Neuroimaging studies have also often used clinical neuropsychological tests of attention (e.g., Stroop task, PASAT) that can have limited construct validity (Spikman et al., 2001) and where there are limited data 
concerning functional neuroanatomy supporting these tests in healthy subjects.

Here we assessed neural activity patterns in patients with moderate-to-severe TBI with evidence of DAI as the primary neuropathology and without significant focal lesions. We used a visual feature integration task designed to measure various attentional processes (Stuss et al., 1989). The task included conditions of increasing complexity and difficulty: a single-feature condition, where subjects had to respond to a Target shape consisting of a specific combination of components and ignore other distracter shapes, requiring only one component to be kept in mind, and a multi-feature condition where subjects could only respond correctly to the Target shape if all three components were kept in mind. The task also included a condition designed to assess focused attention, in which subjects avoided potential distraction by redundant information. TBI patients previously studied had slower reaction times (RTs) in both single- and multi-feature conditions of the task compared to controls (Stuss et al., 1989). In addition, TBI patients demonstrated more variability in RT across all three conditions than in comparison subjects (Stuss et al., 1994). Thus, one major advantage to this study is the use of an experimentally validated test to examine the neural correlates of attention in TBI (Stuss et al., 2002). Given the diffuse neuropathology of our patient sample, we predicted increases in distributed alterations in neural activity following TBI in association with performance of this task, particularly in prefrontal regions. We used a multivariate neuroimage analysis method, partial least squares (PLS; McIntosh et al., 2004), which is more sensitive to such distributed effects than traditional univariate neuroimaging analysis approaches as it emphasizes the interdependencies among neural regions.

\section{MATERIALS AND METHODS SUBJECTS}

Eight patients (four males) with moderate-to-severe TBI were recruited. All subjects were right-handed, native English speakers and were screened for previous neurological injury, major medical conditions affecting cognition, and history of psychiatric illness. Three sets of comparison subjects were used. Ten healthy comparison subjects (three males) were recruited for functional imaging comparison; two other groups helped characterize TBI patients' neuropsychological status and structural neuroimaging data (see Table 1).

Traumatic brain injury patients were recruited from consecutive admissions as part of the Toronto TBI study (Fujiwara et al., 2008; Levine et al., 2008; Turner and Levine, 2008). All patients had sustained a TBI as a result of a motor vehicle accident and were in the chronic stage of recovery at the time of study participation (see Table 1). Despite their significant TBIs, all patients demonstrated good functional recovery as evidenced by a return to pre-injury employment or academic status. Injury severity was determined by GCS as documented by the trauma team leader's score at the time of discharge from the Trauma Unit, corresponding to the

Table 1 | Traumatic brain injury patient demographics, acute injury characteristics, structural neuroimaging data, and neuropsychological test data.

\begin{tabular}{|c|c|c|c|c|c|c|c|c|c|c|}
\hline Subject no. & 1047 & 3646 & 3651 & 1054 & 3652 & 3639 & 3649 & 3640 & Patient means (SD) & Control means (SD) \\
\hline \multicolumn{11}{|l|}{ DEMOGRAPHICS } \\
\hline Age (years) & 47 & 28 & 26 & 26 & 24 & 31 & 30 & 20 & $31.5(7.2)$ & $27(5)$ \\
\hline Education (years) & 16 & 16 & 18 & 17 & 17 & 15 & 16 & 13 & $16.8(1.2)$ & $\mathrm{n} / \mathrm{a}$ \\
\hline \multicolumn{11}{|l|}{ INJURY CHARACTERISTICS } \\
\hline Glasgow coma score & 14 & 6 & 3 & 9 & 7.5 & 9 & 10.5 & 13 & $8.4(3.4)$ & \\
\hline Loss of consciousness (h) & 0.017 & 96 & 120 & 26 & $\mathrm{n} / \mathrm{a}$ & 5 & 168 & 60 & $142.1(154.1)$ & \\
\hline Post-traumatic amnesia (h) & 168 & 1080 & 1176 & 240 & 504 & 504 & $\mathrm{n} / \mathrm{a}$ & 504 & $669(414.0)$ & \\
\hline Time since injury (years) & 3.7 & 2.7 & 1.9 & 3.7 & 2.1 & 2.0 & 2.7 & 3.1 & $2.73(0.72)$ & \\
\hline Severity classification & Mod. & Sev. & Sev. & Sev. & Sev. & Mod. & Mod. & Sev. & & \\
\hline \multicolumn{11}{|c|}{ QUANTITATIVE STRUCTURAL IMAGING $\left(\mu L \times 10^{4}\right)$} \\
\hline Gray matter volume & 53.4 & 60 & 60.8 & 61.4 & 62.6 & 61.8 & 62 & 63.7 & $59.9(3.1)$ & $63.1(1.6)$ \\
\hline White matter volume & 42.8 & 42.4 & 43.2 & 43.3 & 42.7 & 41.5 & 41.5 & 45.2 & $42.75(1.0)$ & $46.8(1.8)$ \\
\hline Total cerebral spinal fluid & 26.9 & 21.2 & 20.1 & 19.2 & 18.6 & 20.7 & 20.6 & 15.6 & $21.2(2.6)$ & $16.3(1.9)$ \\
\hline \multicolumn{11}{|c|}{ SELECT NEUROPSYCHOLOGICALTESTS } \\
\hline Shipley institute of living scale (verbal) & 34 & 36 & 34 & 28 & 29 & 34 & 29 & 24 & $32.4(3.2)$ & $30(4)$ \\
\hline Verbal fluency (\# of words generated) & 47 & 33 & 53 & 49 & 51 & 35 & 45 & 42 & $44.1(7.4)$ & $40(10)$ \\
\hline Symbol-digit, written (\# correct) & 58 & 45 & 61 & 69 & 56 & 51 & 69 & 51 & $59.3(8.5)$ & $59(10)$ \\
\hline Symbol-digit, oral (\# correct) & 66 & 49 & 83 & 88 & 67 & 64 & 88 & 67 & $71.3(13.8)$ & $72(12)$ \\
\hline Trail-making test (BA) & 41 & 25 & 10 & 25 & 21 & 12 & 25 & 36 & $23.5(9.7)$ & $31(15)$ \\
\hline Wisconsin card test (perseverations) & 16 & 14 & 10 & 11 & 10 & 13 & 11 & 18 & $11.9(2.2)$ & $20(9)$ \\
\hline Self-ordered pointing (total) & 7 & 8 & 4 & 4 & 3 & 6 & 4 & 11 & $4.6(2.3)$ & $6(4)$ \\
\hline
\end{tabular}

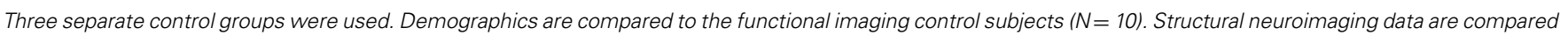
to age-matched healthy adults ( $N=12$; Levine et al., 2008). Neuropsychological data are compared to age-, education-, and socioeconomic-matched healthy adults ( $N=27$; Turner and Levine, 2008). Data from all three groups are included in the column "Control" in the table. 
recommended 6-h GCS score (Teasdale and Jennett, 1974). Severity in one case (1054) was upgraded from that indicated by the GCS due to extended post-traumatic amnesia. Seven of the eight patients underwent a separate structural MRI protocol (for more details see Levine et al., 2008). Interpretation by a board-certified radiologist specializing in TBI indicated evidence of DAI-related neuropathology (hemosiderin deposits) localized to the frontal lobes (six patients), parietal lobes (four patients), the temporal lobe (two patient), the corpus callosum (two patients), the basal ganglia (one patient), and the thalamus (one patient). No patient had lesions greater than $3 \mathrm{~mm}$ in diameter, except patient 1047 who had a lesion in the $\mathrm{R}$ temporal pole, with a volume of $0.713 \mu \mathrm{L} \times 10^{4}$. This patient was retained in the sample as the lesion was relatively small and in a region remote from areas crucial to processing of the feature integration task. Whole-brain volumetric measures of gray matter, white matter, and CSF following our published image analysis methods (Levine et al., 2008) showed evidence of atrophy relative to age- and education-matched comparison subjects. Taken together, the radiological interpretation and significant volume loss in the TBI patients are consistent with DAI.

All eight patients underwent neuropsychological testing. Neuropsychological test data were compared with a local normative sample of age, education, and socioeconomically matched control subjects. TBI patients demonstrated average to high average performance on the verbal subtask of the Shipley Institute of Living Scale (Zachary, 1986). TBI patients also performed normally on other neuropsychological tests of attention and executive functioning, including (with one exception) a task explicitly measuring executive control within working memory.

\section{VISUAL FEATURE-MATCHING TASK}

The experimental design was based on a previous behavioral study that examined the relationship between RT and varying degrees of complexity on a feature integration task in subjects with head injury (Stuss et al., 1989).

Subjects were shown geometric shapes while scanning during one of three conditions. In each condition, the stimuli were presented at random and considered either "Target" or "Non-target." Subjects were instructed to press one button to the Target stimulus and a different button to the Non-target stimulus (see below for details). The stimuli had three different components (shape, color, and line orientation within the shape), and each of these could appear in one of four possible states (see Figure 1 for examples):

(1) Single-Feature Condition: one of the four geometric shapes selected as the Target was presented randomly. The remaining three shapes were the Non-targets. Both Target and Nontarget shapes shared the same color and line orientation components so that the stimuli were complex, but subjects had to make their choice based solely on shape.

(2) Multi-Feature Condition: the Target possessed one randomly selected combination of components. All other stimuli were Non-targets.

(3) Redundant Condition: the stimuli were characterized by the same components as in the multi-feature and single-feature conditions, but no state specific to the Target could ever appear

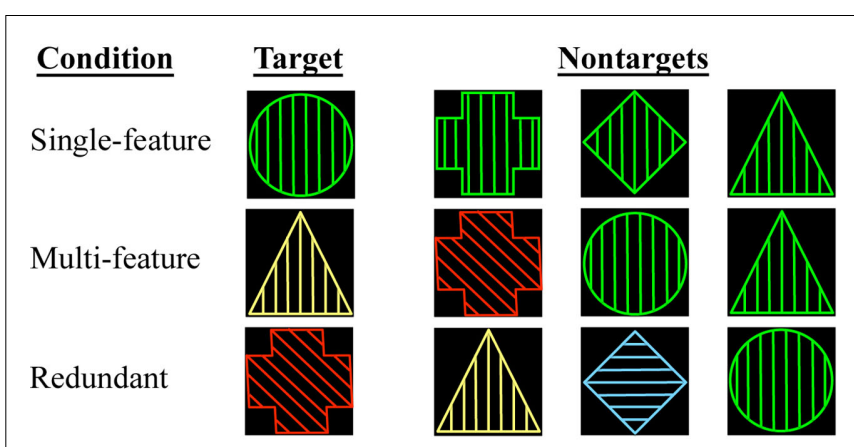

FIGURE 1 | Depiction of the single-feature, multi-feature, and redundant reaction time conditions. For each condition, examples of a Target and of Non-targets are shown where relevant.

in the Non-target. For example if the Target was a red circle with vertical lines, no Non-target appeared as red, a circle, or with vertical lines. Hence the stimuli were as complex as in the single- and multi-feature conditions, but most of the information was redundant.

Stimuli were intermixed with visual fixation on a cross, which was taken as the baseline condition for brain activity. Stimuli were presented in blocks organized by condition. The timing of the stimuli corresponded to an event-related design so that the hemodynamic response function (HRF) could be optimally separated out for each stimulus.

Stimuli were presented in the following manner: participants rested their index and middle fingers on a two-button pad. At the beginning of each recording block, a Target stimulus was presented for $6 \mathrm{~s}$. Four seconds after the Target stimulus disappeared, a series of 48 test stimuli were presented at inter-stimulus intervals varying between 2.5 and $7.5 \mathrm{~s}$. Each test stimulus remained on screen for $2 \mathrm{~s}$ or until a response was made. Twenty-five percent of the test stimuli matched the Target in each block. Thus, there were a total of 36 Target trials and 108 Non-target trials in each condition. In addition, there were a total of 72 visual fixation baseline trials.

Reaction time measurements were taken for each trial. Mean RT and variability [defined as coefficient of variation (SD/mean), CVRT] measures were calculated for each condition for the Nontarget trials. Non-target trials were analyzed separately as they required more complex attentional processing demands than the Target trials. By definition, the Target trials match on all possible features, whereas the Non-target trials have to be analyzed on a feature-by-feature basis to determine the match. In addition, because the Non-target trials share features with the Targets in the single- and multi-feature conditions, correct response to the Non-targets requires inhibition of the response to the Target which may be triggered by these shared features. As there were only 36 Target trials, these were not analyzed separately due to lack of power. Control and TBI groups were compared on mean RT, CVRT, and accuracy on the task using independent samples $t$-tests. RT measurements were not available for one patient and one control subject due to technical difficulties. 


\section{fMRI SCANNING AND ANALYSES}

Scanning was performed on a 3.0-T MRI system (Signa 3T94 hardware, VH3M3 software; General Electric Healthcare, Waukesha, WI, USA). Before functional scanning, standard high-resolution three-dimensional T1-weighted fast spoiled gradient (FSPGR) echo pulse sequence was used to acquire 124 axial anatomic MRI slices. Functional scans were obtained using a single-shot T2*-weighted pulse sequence with spiral in-out, achieving 26 axial slices (TR/TE of $2000 / 30 \mathrm{~ms}$, flip angle of $70^{\circ}$, acquisition matrix $=64 \times 64$, FOV of $20 \mathrm{~cm}$, and $3.1 \mathrm{~mm} \times 3.1 \mathrm{~mm} \times 5 \mathrm{~mm}$ voxel resolution).

Images were preprocessed using the analysis of functional neuroimages (AFNI; Cox, 1996) and Statistical Parametric Mapping (SPM99) software (Wellcome Department of Cognitive Neurology, London, UK). The functional time series was "despiked," and six parameter rigid body motion correction was done by coregistering volumes to a reference functional scan using AFNI (Cox and Jesmanowicz, 1999). The difference in the timing of slice acquisition was also corrected for. Subsequently, using SPM99, images were spatially transformed to standard MNI space (Evans et al., 1992) using a linear transformation with sinc interpolation only. The resulting voxel size was $4 \mathrm{~mm} \times 4 \mathrm{~mm} \times 4 \mathrm{~mm}$. The data were then smoothed spatially with an 8 -mm full-width half- maximum Gaussian filter. The effect of any global differences in fMRI signal intensity between individual subjects was removed by calculating the percentage signal change for each voxel during the active conditions as compared to the signal intensity during visual fixation for each subject within each run.

The preprocessed fMRI data were then analyzed using a PLS approach (McIntosh and Lobaugh, 2004). Specifically, PLS is a set of multivariate analysis techniques that can be used to identify a new set of variables (called latent variables, LVs) that relate any set of independent measures, such as the experimental design (as in this study) or activity in a seed region, to a set of dependent measures, in this case, brain activity of each subject group (McIntosh et al., 1996). PLS carries out the computation of the optimal least squares fit to cross-block correlation between the independent and dependent measures. In comparison to principal component analysis (PCA), PLS has the advantage that solutions are constrained to the relevant experimental manipulations (McIntosh and Lobaugh, 2004). Relative to a more traditional general linear model (GLM) analysis, PLS is more powerful to detect distributed changes because it analyzes the patterns of covariance across all voxels simultaneously rather than assessing each voxel's activity as independent from the rest of the brain's activity. In addition, PLS uses a bootstrapping estimation technique that emphasizes the reliability of voxel activation over signal strength alone, which allows the inclusion of consistently activating voxels, which may have been excluded in the GLM analysis into the overall brain activity pattern. PLS data are interpreted by relating the pattern of reliably active voxels to a set of brain scores, which are determined for each task condition and subject group. The brain scores are similar to the factor scores in a PCA and indicate how strongly the subject groups express the brain activity pattern. If, for example, the brain score for a certain condition is positive, then the subject group expresses the pattern reliably for that condition in all voxels that have positive values above threshold. Through this technique, we were able to assess changes in distributed activity occurring after the diffuse injuries caused by TBI.

Only data for Non-target trials were analyzed for the reasons specified above. All the three task conditions (single-feature, multifeature, and redundant) and baseline were included in this analysis. For each condition, the HRF of each voxel was defined as the intensity difference from trial onset during seven consecutive poststimulus temporal lags (lag $=2 \mathrm{~s}$ or $1 \mathrm{TR}$ ) averaged across trials. No assumption was made about the shape of HRF, allowing investigation of changes in task-related activity at different lags along the whole temporal window of $14 \mathrm{~s}$. The data matrix containing all voxels and associated activity values for the seven consecutive poststimulus temporal lags (columns) for all conditions and subjects in each group (rows) was mean-centered column-wise with respect to overall grand average. Singular value decomposition (SVD) was then applied to this matrix to generate mutually orthogonal LVs, with decreasing order of magnitude, analogous to PCA. An LV can be thought of as the optimal values linking the brain activity data with the task design. Each LV consisted of: (i) a singular value, (ii) a pattern of design scores, which provided a set of contrasts across task conditions, and (iii) a singular image, which showed how the spatial distribution of activity across the brain relates to the task conditions and subject groups.

The weighted linear combinations that related the brain activity measurements to group and task design were the "salience" values, which reflected how strongly each given voxel contributed to an LV. A brain score was generated for each subject by multiplying the salience values by the raw voxel data. This reflected how much a subject expressed the pattern of salience values across voxels and temporal lags. Similarly, salience values were generated for the design, which reflected how strongly each task condition contributed to the LV. Design scores were generated in a similar fashion as the brain scores using the design saliences. They can be thought of as a set of contrasts that code the effects resulting from the SVD.

Interpretation of the relation between the polarity of the saliences in the singular image and the direction of HRF change in the areas reliably activated in each LV requires relating the saliences to the group brain scores. For instance, positive saliences would indicate areas that are relatively more active in conditions with positive brain scores. Conversely, negative saliences would indicate areas that are relatively more active in conditions with negative brain scores (see Figure 3 below, for an example).

The significance for each LV as a whole was determined by 500 permutation tests (Edgington, 1980), in which the observations were randomly reordered without replacement to calculate the probability of each LV having occurred by chance. The stability of each voxel's contribution to the latent variable was determined through bootstrap resampling (Sampson et al., 1989; subjects were re-sampled 200 times). Voxels were considered reliable if they had a ratio of salience value to SE (hereafter referred to as the bootstrap ratio, which can be interpreted similarly to a $Z$ score if the distribution for a given voxel is normal; Efron and Tibshirani, 1986). For each lag, clusters with at least 50 contiguous voxels with a bootstrap ratio $\geq+2.5$ or at least 250 contiguous voxels 
with a bootstrap ratio $\leq-2.5$ (equivalent to a confidence interval of $99 \%$ ) were considered to be reliable. As will be seen in the results, the asymmetric cluster size thresholds for positive and negative bootstrap ratios were dictated by large group differences in activity patterns.

Coordinates of the voxel with the peak bootstrap ratio within each cluster were obtained in MNI space. The approximate gyral locations and Brodmann areas were then identified using the Talairach Daemon tool (Lancaster et al., 2000).

We ran two supplementary PLS analyses examining the relationship between brain activity across voxels and experimental conditions separately in both control and TBI patient groups. Reliability and significance were assessed in the same way as the overall PLS analysis described above. We then computed the intersection between each individual group analysis with the overall analysis by identifying voxels that had robust activity (defined by a bootstrap ratio \pm 2.5$)$ in both analyses. This allowed us to identify voxels in which there was a convergence of activity in one subject group with the activity in the overall analysis and helped determine which subject group was driving the overall activity pattern.

Supplementary analyses were done on the hemodynamic response signal obtained from selected clusters of activity that were identified by the Task PLS to confirm the effects obtained between groups and across task conditions. Two-way analysis of variances (ANOVA) looking at the effects of group and task condition were conducted on clusters with both positive and negative saliences. Subsequent simple effects analyses were then conducted.

\section{RESULTS}

Behaviorally, TBI patients and controls differed significantly on Mean RT for all three task conditions [single-feature: $F(1,14)=11.893, p<0.01$, Cohen's $d=-1.75$; multi-feature: $F(1,14)=23.230, \quad p<0.001$, Cohen's $d=-2.40$; redundant: $F(1,14)=5.065, p<0.05$, Cohen's $d=-1.12]$. For CVRT, there was a significant group difference for the multi-feature condition $[F(1,14)=7.529, p<0.05$, Cohen's $d=-1.41]$. The group difference in CVRT fell just short of statistical significance for the singlefeature condition $[F(1,14)=4.290, p=0.057$ Cohen's $d=-1.09]$. There was no significant difference for the redundant condition $[F(1,14)=1.901, p=$ n.s., Cohen's $d=-0.66]$. Finally, there was a significant group difference in accuracy on the multi-feature condition $[F(1,14)=5.134, p<0.05$, Cohen's $d=1.08]$, but not the single-feature $[F(1,14)<1, p=$ n.s., Cohen's $d=0.29]$ or redundant conditions $[F(1,14)=2.418, p=$ n.s., Cohen's $d=0.73]$. See Figure 2 for the group behavioral results.

The Task PLS analysis on the fMRI data revealed one significant LV (explained cross-block covariance $=29.94 \%, p<0.024$ ). The group brain scores with $95 \%$ confidence intervals for the LV are shown in Figure 3A. The brain scores are considered reliable if the confidence interval does not cross 0 . The LV showed similar activity patterns for TBI patients and controls, but these activity patterns were expressed for different conditions by each group: the multi-feature condition for controls and the singlefeature condition and baseline in TBI patients. The LV also showed additional activity patterns in TBI patients for the multi-feature and redundant conditions. The clusters with positive and negative saliences are listed in Table 2, respectively, and are shown in
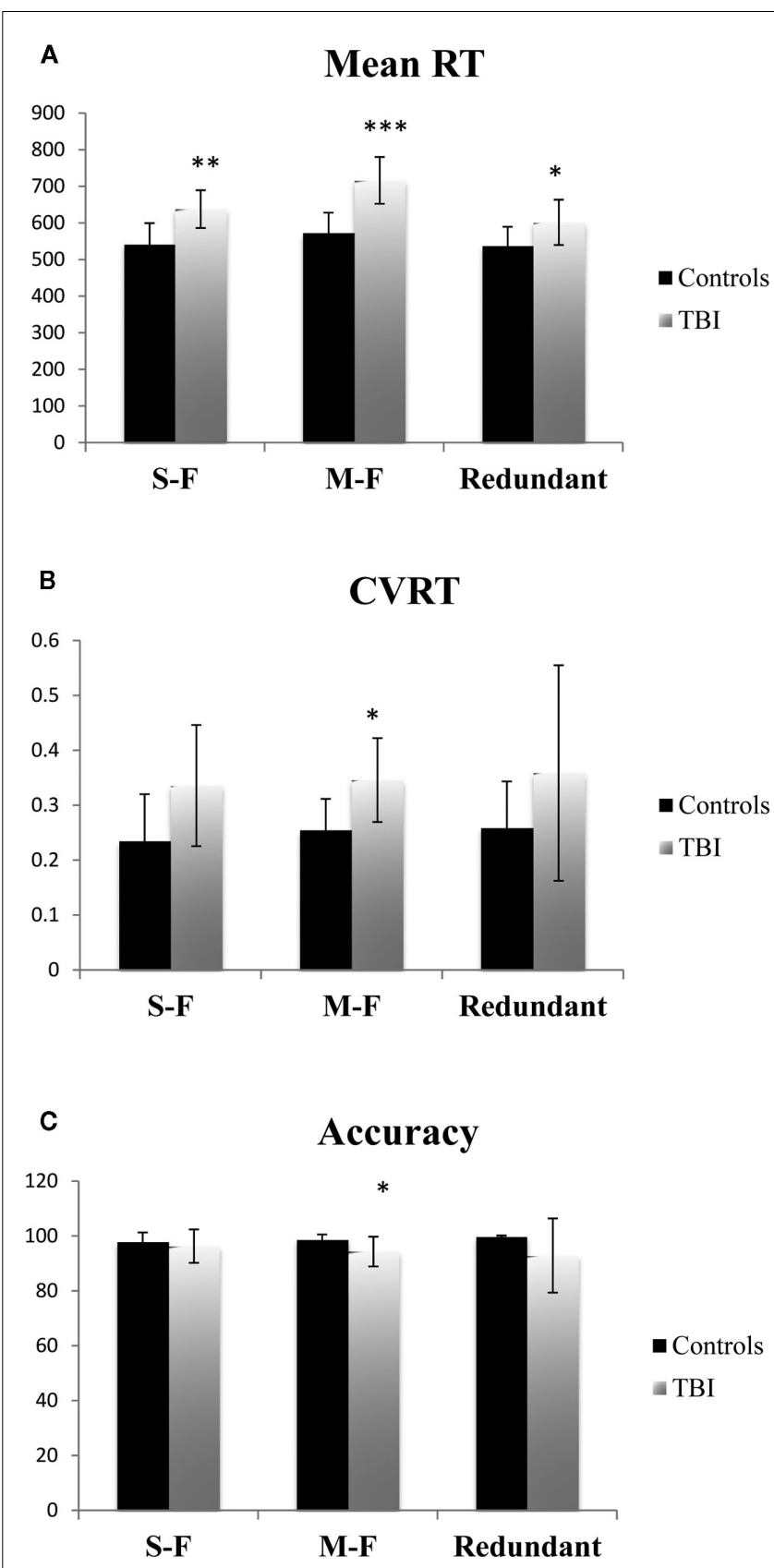

FIGURE 2 | Mean reaction time (RT) (A), coefficient of variation on reaction time (CVRT) (B), and (C) accuracy for each task condition for functional imaging control subjects and TBI patients. Error bars indicate SE of the mean. ${ }^{*}=$ Significant differences between controls and TBI $(p<0.05) .{ }^{*}=$ Significant differences between control and TBI $(p<0.01)$.

Figure 3B. Regions with positive saliences show greater activity for the multi-feature condition in controls and the single-feature condition and baseline in TBI patients. These regions included cerebellum, thalamus, ventral premotor cortex, middle and posterior cingulate, dorsolateral prefrontal cortex (DLPFC), anterior prefrontal cortex (aPFC), and the parahippocampal gyrus. Regions with negative saliences show greater activity for the multi-feature 


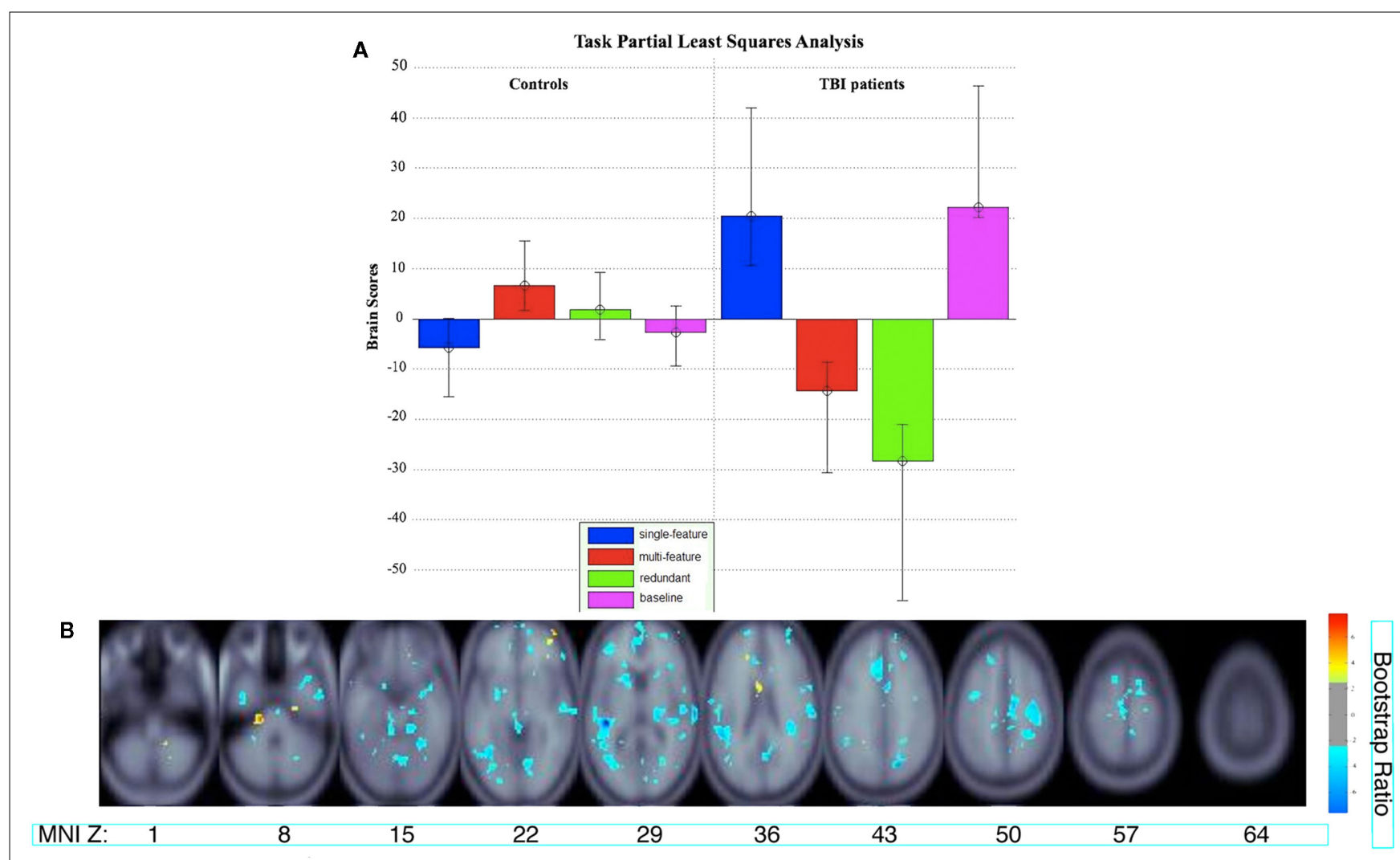

FIGURE 3 | (A) Brain scores for the significant latent variable (LV) from the task PLS analysis. Error bars indicate confidence intervals. The average brain scores for each group and condition are considered to reliably contribute to the LV if the confidence interval does not cross 0 . The brain scores are similar to factor scores and indicate how strongly an individual subject expresses the patterns on the latent variable. The score is the dot product of the subject's raw brain activity data and the singular image from the latent variable showing the pattern of brain activity that is most related to the group and task differences (Mclntosh et al., 2004). (B) Singular image showing reliable clusters (number of voxels $\geq 50$ for positive saliences, number of voxels $\geq 250$ for negative saliences, bootstrap ratio $\geq \pm 2.5$ ). The brain activity patterns occur five lags (10 s) after stimulus onset time. The $X$ axis shows the location of the axial slice with a coordinate along the $Z$ axis in $\mathrm{MNI}$ atlas space. Warm colors indicate clusters with positive bootstrap ratios, which were more active in group and task conditions with positive brain scores in (A). Cool colors indicate clusters with negative bootstrap ratios, which were more active for group and task conditions with negative brain scores. Letter designations serve as a cross-reference to regions listed in Table 2. The bootstrap ratio map is superimposed on the average anatomical scans from all 18 subjects. and redundant conditions in TBI patients only and included a widespread set of regions in frontal, parietal, and occipital cortices.

See Figures 4A,B for the results of the two-way ANOVAs on the signal of selected voxels identified by the Task PLS.

The supplementary individual group Task PLS analyses did not reveal a significant LV $(p>0.05)$ for either control or TBI patient groups. Qualitative inspection of the intersection between the control Task PLS and the overall analysis revealed almost no overlap in activity patterns (Figure 5A). However there was overlap between TBI brain activity and the overall analysis in several regions including two large clusters in the left inferior parietal lobule (Figure 5B).

\section{DISCUSSION}

Our findings demonstrate differentiated functional neural activity underlying attentional processes in subjects with DAI neuropathology due to TBI compared to neurologically normal individuals. Previous studies examining executive control processes such as working memory and attention after TBI have been confounded by the fact that heterogeneous neuropathology subtypes were included in their samples (Kim et al., 1999; Christodoulou et al., 2001; Perlstein et al., 2004; McAllister et al., 2006). In the present study, focal lesions were restricted to small hemosiderin deposits spread throughout the cortex, which reflected the presence of DAI, except in the case of one subject who had a small contusion in an area remote from regions supporting attentional processes. On the whole, the subjects in our sample showed a predominance of DAI as the underlying neuropathology without the involvement of focal lesions. Evidence of DAI is additionally found in the fact that our patients show whole-brain parenchymal (gray + white matter) volume loss compared to controls. The relatively pure neuropathology of our sample allowed us to draw more specific conclusions about the effect of DAI on neural activity supporting attention than has been previously possible.

Previous studies with TBI subjects have shown activity changes that occur on a distributed level (Christodoulou et al., 2001; McAllister et al., 2006; Kim et al., 2008; Levine et al., 2008; Turner and 
Table 2 | Reliable clusters identified for the significant LV in the task PLS analysis (bootstrap ratios $\geq \pm 2.5$ ).

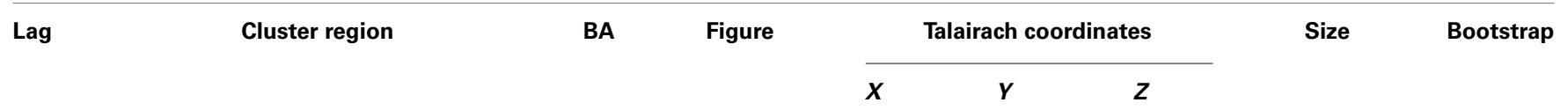

\begin{tabular}{|c|c|c|c|c|c|c|c|c|c|}
\hline \multicolumn{10}{|c|}{ A. POSITIVE SALIENCES/BOOTSTRAP RATIO } \\
\hline 1 & $\mathrm{~L}$ & Thalamus & & & -22 & -34 & 11 & 213 & 4.32 \\
\hline 1 & $\mathrm{R}$ & Precentral gyrus & 44 & & 44 & 14 & 10 & 183 & 5.41 \\
\hline 1 & $\mathrm{R}$ & Posterior cingulate & 29 & & 12 & -46 & 15 & 147 & 6.37 \\
\hline 1 & $\mathrm{R}$ & Cerebellar tonsil & & & 30 & -49 & -40 & 140 & 5.77 \\
\hline 1 & $\mathrm{R}$ & Inferior frontal gyrus & 47 & & 28 & 35 & 0 & 120 & 4.95 \\
\hline 2 & $\mathrm{R}$ & Cingulate gyrus & 31 & & 24 & -23 & 36 & 65 & 4.44 \\
\hline 2 & $\mathrm{R}$ & Cerebellar tonsil & & & 24 & -62 & -39 & 50 & 3.75 \\
\hline 3 & $\mathrm{~L}$ & Inferior frontal gyrus & 46 & & -42 & 35 & 2 & 94 & 6.21 \\
\hline 5 & $\mathrm{~L}$ & Culmen & & a & -26 & -27 & -34 & 93 & 7.67 \\
\hline 5 & $\mathrm{R}$ & Middle frontal gyrus & 10 & $b$ & 42 & 64 & -9 & 73 & 6.58 \\
\hline \multicolumn{10}{|c|}{ B. NEGATIVE SALIENCES/BOOTSTRAP RATIOS } \\
\hline 1 & $\mathrm{~L}$ & Red nucleus & & & 0 & -22 & -4 & 357 & -6.44 \\
\hline 2 & $\mathrm{~L}$ & Middle temporal gyrus & 22 & & -55 & -41 & 2 & 1570 & -6.82 \\
\hline 2 & $\mathrm{~L}$ & Precentral gyrus & 6 & & -48 & 2 & 31 & 1299 & -6.48 \\
\hline 2 & $\mathrm{R}$ & Middle frontal gyrus & 46 & & 50 & 21 & 23 & 963 & -5.56 \\
\hline 2 & $\mathrm{R}$ & Culmen & & & 10 & -68 & -6 & 798 & -6.98 \\
\hline 2 & $\mathrm{~L}$ & Subthalamic nucleus & & & -8 & -14 & -3 & 739 & -5.89 \\
\hline 2 & $L$ & Postcentral gyrus & 3 & & -30 & -35 & 44 & 684 & -5.58 \\
\hline 2 & $\mathrm{R}$ & Superior temporal gyrus & 22 & & 46 & -17 & 9 & 667 & -4.93 \\
\hline 2 & $\mathrm{~L}$ & Superior frontal gyrus & 10 & & -18 & 61 & 17 & 624 & -6.32 \\
\hline 2 & $\mathrm{~L}$ & Cingulate gyrus & 24 & & -6 & 19 & 29 & 531 & -4.66 \\
\hline 3 & $\mathrm{~L}$ & Putamen & & & -28 & 0 & 6 & 567 & -6.66 \\
\hline 3 & $\mathrm{~L}$ & Precuneus & 31 & & -30 & -71 & 20 & 393 & -4.89 \\
\hline 3 & $\mathrm{R}$ & Precentral gyrus & 6 & & 50 & 3 & 20 & 362 & -4.89 \\
\hline 4 & $\mathrm{~L}$ & Cingulate gyrus & 24 & & -16 & 2 & 46 & 613 & -5.88 \\
\hline 4 & $\mathrm{R}$ & Medial frontal gyrus & 6 & & 16 & 5 & 61 & 497 & -4.64 \\
\hline 4 & $\mathrm{~L}$ & Insula & 13 & & -38 & -44 & 22 & 443 & -7.66 \\
\hline 4 & $\mathrm{~L}$ & Precentral gyrus & 4 & & -58 & -12 & 26 & 357 & -6.69 \\
\hline 5 & $\mathrm{~L}$ & Cingulate gyrus & 32 & $d$ & -8 & 17 & 36 & 2197 & -7.82 \\
\hline 5 & $\mathrm{~L}$ & Superior temporal gyrus & 41 & e & -46 & -29 & 7 & 1822 & -13.20 \\
\hline 5 & $\mathrm{R}$ & Superior temporal gyrus & 42 & $f$ & 69 & -19 & 6 & 1108 & -7.73 \\
\hline 5 & $\mathrm{~L}$ & Amygdala & & $g$ & -20 & -12 & -10 & 753 & -4.33 \\
\hline 5 & $\mathrm{R}$ & Precentral gyrus & 6 & $\mathrm{~h}$ & 38 & -12 & 41 & 742 & -6.48 \\
\hline 5 & $\mathrm{~L}$ & Medial frontal gyrus & 10 & i & 0 & 66 & -3 & 646 & -6.75 \\
\hline 5 & $\mathrm{~L}$ & Inferior temporal gyrus & 37 & j & -59 & -56 & -2 & 429 & -5.40 \\
\hline 5 & $\mathrm{R}$ & Uncus & 28 & k & 28 & 1 & -28 & 390 & -7.72 \\
\hline
\end{tabular}


Table 2 | Continued

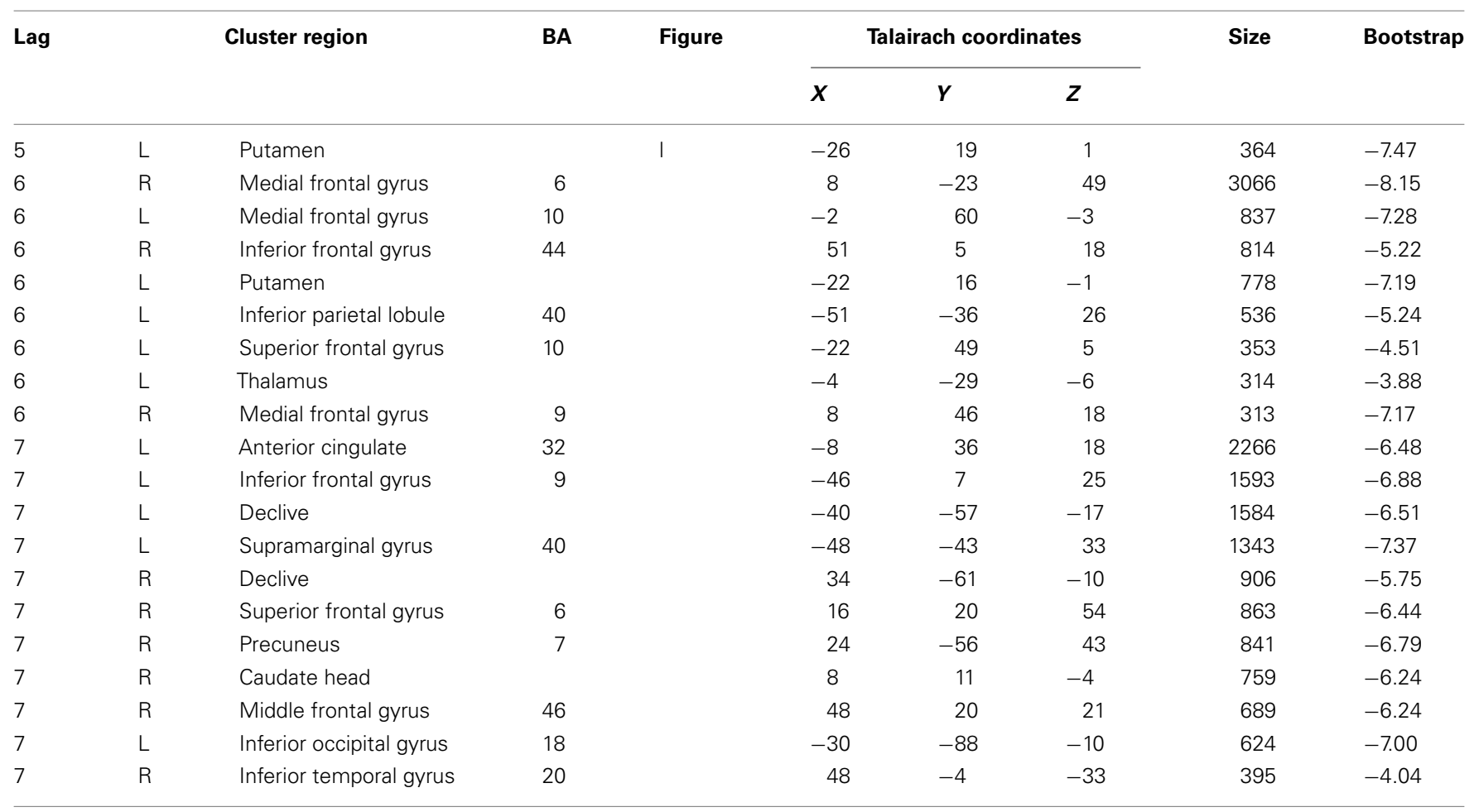

Lag refers to the time period, in TRs of $2 \mathrm{~s}$ each, after stimulus onset during which the peak bootstrap ratio occurred. Cluster region and BA indicate the locations and Brodmann areas determined from Talairach and Tournoux (1988). A designation for cross-reference to regions active in lag 5 and appearing in Figure $\mathbf{3}$ is indicated. The letter designation appears to the right of the region in Figure 3. Talairach coordinates $x, y$, and $z$ indicate voxel coordinates in Talairach space. Size refers to the number of contiguous voxels in the cluster. Bootstrap is an index of reliability across subjects.

Levine, 2008). Some studies have identified altered patterns of activity compared to controls (Levine et al., 2002; Kim et al., 2008) whereas other studies have shown load-related activity differences in TBI patients in areas devoted to task processing that are also active in controls (Perlstein et al., 2004). In the present study, we demonstrate an overlapping set of regions that are active for the task in both TBI patients and controls; however, we also find a set of regions that are uniquely recruited by TBI patients for the more visually complex task conditions. TBI patients and controls both show activity in a set of regions composed of cerebellum, thalamus, ventral premotor cortex, middle and posterior cingulate, DLPFC, aPFC, and the parahippocampal gyrus. These regions have been shown to constitute large-scale networks involved in cognitive control and maintenance of task-relevant information, as well as play a role in spatial attention (Kim et al., 1999). A host of studies have ascribed top-down attentional control to the DLPFC (e.g., MacDonald et al., 2000; Koechlin et al., 2003; Stuss and Alexander, 2007). More recent functional connectivity work has also implicated middle cingulate/precuneus as comprising a network including DLPFC that is involved in task-related control (Dosenbach et al., 2007). In addition, the posterior cingulate is related to the speed of spatial target detection (Mesulam et al., 2001). Posterior cingulate/retrosplenial cortex has extensive anatomical connections to neocortical and hippocampal regions (Pandya et al., 1981), and therefore may show greater activity during attention to events that have acquired salience through experience. Several $\mathrm{fMRI}$ studies have shown the aPFC and frontal operculum to be involved in maintenance of task sets (Braver et al., 2003; Dosenbach et al., 2006; Sakai and Passingham, 2006), which may be more generally related to the role of these regions in working memory (Wager and Smith, 2003). More recent work has described a cingulo-opercular network that includes the thalamus and aPFC that shows sustained activity for the maintenance of task goals (Dosenbach et al., 2007). Both networks related to top-down control have been shown to have connections with the cerebellum (Dosenbach et al., 2008), which is important in error monitoring (Fiez, 1996). It has also been recently shown that TBI patients with increased severity as indicated by GCS score show increased activity in cingulate and thalamic structures during a task requiring cognitive control (Scheibel et al., 2009); however, these data were taken from TBI patients in the acute phase of recovery. In addition, it has been shown that older participants have augmented activity in regions related to cognitive control during more complex task processing demands (Vallesi et al., 2011), which may reflect some commonalities in neural reorganization in neuropathologies causing damage that is diffuse in nature.

Our results demonstrate that activity in regions thought to mediate cognitive control persists into the chronic stage of TBI for relatively moderate task demands. We did not find activity in the anterior cingulate and inferior parietal regions that are often associated with attentional control networks. This may be due to the fact that the analysis only identified overlapping clusters in 

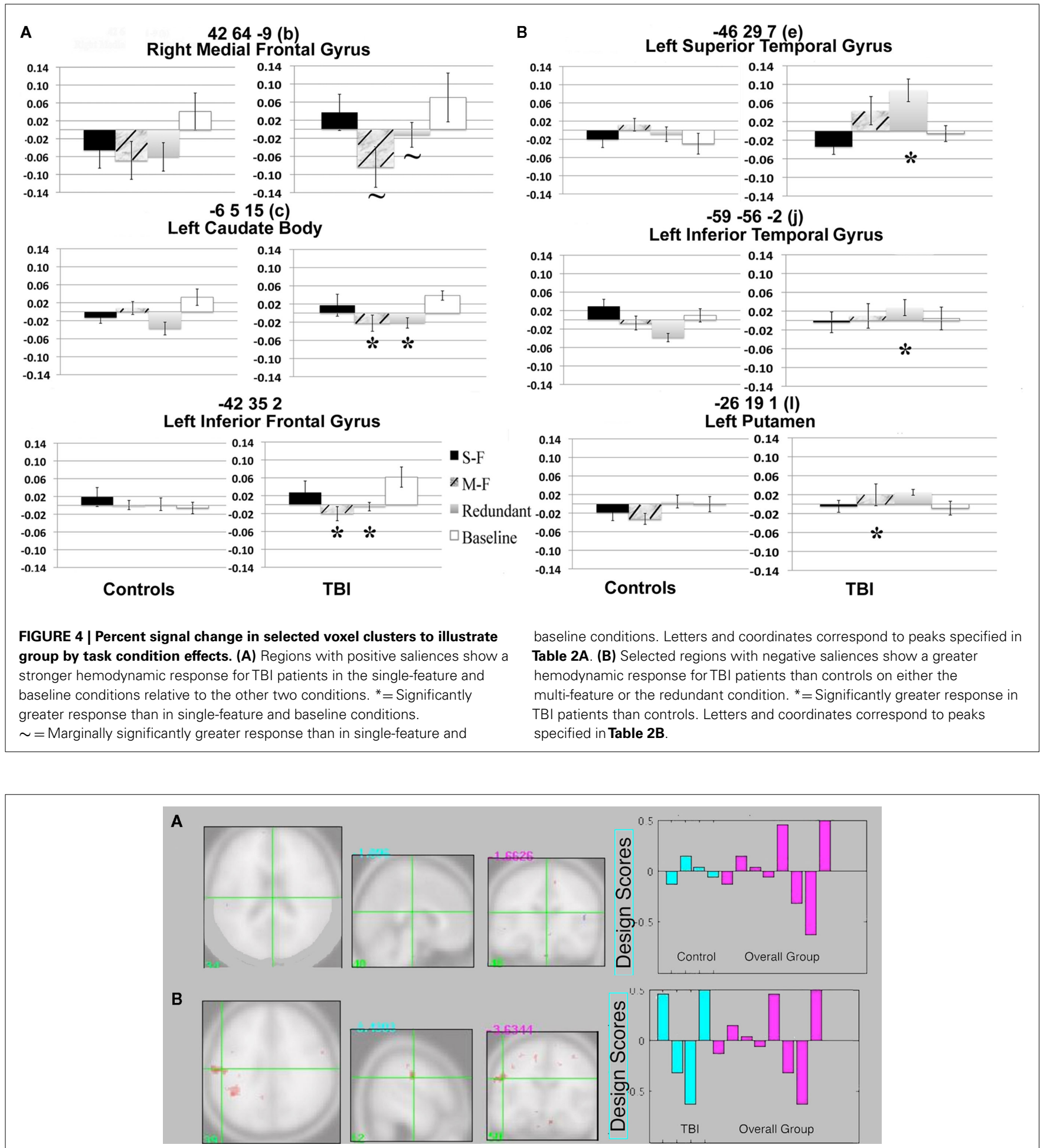

FIGURE 5 | (A) Intersection between control Task PLS analysis and overall group analysis. There is very little overlap in brain activity patterns. (B) Intersection between TBI Task PLS analysis and overall group analysis. Several regions show overlapping activity, including two larger clusters in the left inferior parietal lobule, suggesting the brain activity patterns in the overall analysis are primarily being driven by the TBI group. Design score plots show pattern from the single group analysis in blue and the overall group analysis in pink (repeated from Figure $\mathbf{3 A}$ ), with design scores for the single-feature, multi-feature, redundant, and baseline conditions shown from left to right for each group. The design scores reflect how much the task condition contributes to the overall latent variable pattern for each group. 
TBI patients and controls, and these regions may show spatially differentiated activity patterns between the subject groups.

We show that these brain activity patterns related to task processing are activated for the multi-feature condition in controls and the single-feature condition in TBI patients. This supports previous findings (McAllister et al., 2001) that brain activity for relatively moderate working memory task demands in TBI patients is similar to processing of increased task demands in control subjects. In McAllister et al. (2001) study, parametrically increasing task load resulted in dose-dependent increases of activity in working memory-related regions for control subjects. However, TBI patients showed augmented activity during moderate processing loads that plateaued as task difficulty increased. Here we demonstrate a similar dose-dependent effect for cognitive control-related activity. Our findings show that control subjects recruit the neural regions described above while integrating three features of the Target shape and maintaining this information throughout the task, whereas TBI patients require the same attentional resources in order to maintain only the shape of the target, which they are able to do with a similar level of accuracy compared to controls (for an analysis of the functional connectivity underlying this pattern in patients with TBI and DAI, some of which also participated in the present study, see Turner et al., 2011). The fact that we were able to show this relationship in areas associated with cognitive control underscores the importance of using a task that is experimentally validated to measure attention as a construct when assessing the neural correlates of TBI.

We additionally show that the TBI participants robustly recruit the same attentional resources involved in the single-feature condition during the baseline condition.

Importantly, this finding may inform previous research showing altered functional activity after TBI (Levine et al., 2002). While this prior study argued that altered functional activity may be due to cortical disinhibition resulting from the axonal deafferentation due to DAI, the results could not easily disambiguate this from the possibility of cortical activity being instead driven by compensatory processes. Our demonstration of inappropriate task-related activity occurring in the absence of a stimulus during the baseline trials appears to support the notion that altered functional activity in TBI is more likely due to cortical dysregulation and an inability to inhibit activity. Furthermore, because the baseline trials were rapidly interspersed between the task trials, the mechanism of cortical dysregulation resulting in task-related activity persisting throughout the baseline may be an overall slowness in processing of all three task conditions for the TBI patients compared to the controls. Although slowness in speed of information processing has been observed behaviorally after TBI (see Mathias and Wheaton, 2007 for a review), there are no neuroimaging studies investigating a related delay in the hemodynamic response returning to baseline, and therefore this remains an open question.

It has been suggested that a lack of focusing of activity after TBI reflects reduced efficiency of neural processing (Levine et al., 2002). Our results demonstrate a widespread set of frontal, parietal, and occipital regions that are uniquely active in TBI patients during the multi-feature and redundant conditions. It is likely that this set of regions identified in TBI patients reflects decreased efficiency during task processing as would also seem to be suggested by the slowed task performance compared to controls. Similar findings of more widespread activity in older participants has also been interpreted as indicative of neural inefficiency in cases when performance was matched to or worse than controls (Morcom et al., 2007; Grady, 2008). Both of these conditions present stimuli of increasing complexity compared to the single-feature condition. In addition, both conditions are more difficult than the singlefeature condition because the multi-feature condition requires subjects to integrate more than one salient feature of the Target shape, and, while the redundant condition task can be performed by only remembering one feature, subjects are required to suppress responses to distracting features of the Non-target shapes. TBI patients often show increased difficulty with focused attention and distractibility (Levine et al., 1998), and this is reflected by the fact that the TBI patients made more errors than controls on both the multi-feature and redundant conditions, although this difference was only significant during the multi-feature conditions.

Our findings support previous studies showing more dispersed activity in TBI patients during tasks of working memory and attention (Christodoulou et al., 2001; Levine et al., 2002; Kim et al., 2008) and strongly underscore the distributed nature of the DAI. It is likely that rehabilitation may be accompanied by a reintegration of regions involved in frontoparietal networks (Chen et al., 2006). Previous research has shown decrease in the volume and degree of activation in inferior and middle frontal and parietal regions accompanying improved performance in TBI patients after cognitive training (Kim et al., 2008). As noted above, this finding may be related to disinhibition due deafferentation of axons as a result of DAI. In the presence of a complex task, however, the possibility of compensatory activity changes related to coping with increasing processing demands cannot be ruled out. Because all of the patients in the sample are in the chronic stage, it is feasible that neuroplastic changes occurred since the time of injury. Indeed, axonal sprouting and synaptogenesis have been shown to take place during recovery (Povlishock, 1992).

Finally, a comparison of the intersection between the individual control Task PLS and the overall group analysis vs. the intersection with the individual TBI Task PLS and the overall group analysis revealed that there was overlap mainly between TBI group activity and the overall analysis. This indicated that the regional patterns we identified were tapping into cognitive processing occurring mainly in the patient group after the injury. It is important to point out that most of the patients in the sample show relatively normal performance on neuropsychological tests of attention, working memory, and executive function. This is a common occurrence despite the fact that many TBI patients complain of having difficulty coping with activities of daily life. The insensitivity of traditional neuropsychological tests to the subjective deficits reported by TBI patients poses a problem in terms of diagnosis and treatment. Detection of altered patterns of neural activity may provide a more fine-tuned measure of decreased organization and efficiency of cognitive processes and may be a beneficial addition to a clinician's standard diagnostic arsenal.

\section{ACKNOWLEDGMENTS}

The study was supported by NIH 1R01 HD42385-01 to Brian Levine. 


\section{REFERENCES}

Braver, T. S., Reynolds, J. R., and Donaldson, D. I. (2003). Neural mechanisms of transient and sustained cognitive control during task switching. Neuron 39, 713-726.

Cabeza, R., Anderson, N. D., Locantore, J. K., and McIntosh, A. R. (2002). Aging gracefully: compensatory brain activity in highperforming older adults. Neuroimage 17, 1394-1402.

Cabeza, R., Daselaar, S. M., Dolcos, F., Prince, S. E., Budde, M., and Nyberg, L. (2004). Taskindependent and task-specific age effects on brain activity during working memory, visual attention, and episodic retrieval. Cereb. Cortex 14, 364-375.

Chen, A. J., Abrams, G. M., and D’Esposito, M. (2006). Functional reintegration of prefrontal neural networks for enhancing recovery after brain injury. J. Head Trauma Rehabil. 21, 107-118.

Christodoulou, C., DeLuca, J., Ricker, J. H., Madigan, N. K., Bly, B. M., Lange, G., Kalnin, A. J., Liu, W. C., Steffener, J., Diamond, B. J., and Ni, A. C. (2001). Functional magnetic resonance imaging of working memory impairment after traumatic brain injury. J. Neurol. Neurosurg. Psychiatr. 71, 161-168.

Cox, R. W. (1996). AFNI: Software for analysis and visualization of functional magnetic resonance images. Comput. Biomed. Res. 29, 162-173.

Cox, R. W., and Jesmanowicz, A. (1999). Real-time 3D image registration for functional MRI. Magn. Reson. Med. 42, 1014-1018.

de Chastelaine, M., Wang, T. H., Minton, B., Muftuler, L. T., and Ruff, M. D. (2011). The effects of age, memory performance, and callosal integrity on the neural correlates of successful associative encoding. Cereb. Cortex 21, 2166-2176.

Dosenbach, N. U., Fair, D. A., Cohen, A. L., Schlaggar, B. L., and Petersen, S. E. (2008). A dual-networks architecture of top-down control. Trends Cogn. Sci. (Regul. Ed.) 12, 99-105.

Dosenbach, N. U., Fair, D. A., Miezin, F. M., Cohen, A. L., Wenger, K. W., Dosenbach, R. A. T., Fox, M. D., Snyder, A. Z., Vincent, J. L., Raichle, M. E., Schlaggar, B. L., and Petersen, S. E. (2007). Distinct brain networks for adaptive and stable task control in humans. Proc. Natl. Acad. Sci. U.S.A. 104, 11073-11078.

Dosenbach, N. U., Visscher, K. M., Palmer, E. D., Miezin, F. M., Wenger, K. K., Khang, H. C., Burgund, E. D., Grimes, A. L., Schlaggar, B. L., and Petersen, S. E. (2006). A core system for the implementation of task sets. Neuron 50, 799-812.

Edgington, E. S. (1980). Randomization Tests. New York: Marcel Dekker.

Efron, B., and Tibshirani, R. (1986). Bootstrap methods for standard errors, confidence intervals, and other measures of statistical accuracy. Stat. Sci. 1, 54-77.

Evans, A. C., Marrett, S., Neelin, P., Collins, L., Worsley, K., Dai, W., Milot, S., Meyer, E., and Bub, D. (1992). Anatomical mapping of functional activation in stereotactic coordinate space. Neuroimage 1, 43-53.

Fiez, J. A. (1996). Cerebellar contributions to cognition. Neuron 16, 13-15.

Fujiwara, E., Schwartz, M. L., Gao, F., Black, S. E., and Levine, B. (2008). Ventral frontal cortex functions and quantified MRI in traumatic brain injure. Neuropsychologia 46, 461-474.

Gentleman, S. M., Roberts, G. W., Gennarelli, T. A., Maxwell, W. L., Adams, J. H., Kerr, S., and Graham, D. I. (1995). Axonal injury: a universal consequence of fatal closed head injury? Acta Neuropathol. 89, 537-543.

Grady, C. L. (2008). Cognitive neuroscience of aging. Ann. N. Y. Acad. Sci. 1124, 127-144.

Huang, C.-M., Polk, T. A., Goh, J. O., and Park, D. C. (2011). Both left and right posterior parietal activations contribute to compensatory processes in normal aging. Neuropsychologia. doi: 10.1016/j.neuropsychologia.2011.10. 022. [Epub ahead of print].

Kim, Y.-H., Gitelman, D. R., Nobre, A. C., Parrish, T. B., LaBar, K. S., and Mesulam, M. M. (1999). The large-scale neural network for spatial attention displays multifunctional overlap but differential asymmetry. Neuroimage 9, 269-277.

Kim, Y.-H., Yoo, W.-K., Ko, M.-H., Park, C.-H., Kim, S. T., and Na, D. L. (2008). Plasticity of the attentional network after brain injury and cognitive rehabilitation. Neurorehabil. Neural. Repair 10, 1-10.

Koechlin, E., Ody, C., and Kouneiher, F. (2003). The architecture of cognitive control in the human prefrontal cortex. Science 302, 1181-1185.

Lancaster, J. L., Woldorff, M. G., Parsons, L. M., Liotti, M., Freitas, C. S., Rainey, L., Kochunov, P. V., Nickerson, D., Mikiten, S. A., and Fox, P. T. (2000). Automated Talairach atlas labels for functional brain mapping. Hum. Brain Mapp. 10, 120-131.

Levine, B., Cabeza, R., McIntosh, A. R., Black, S. E., Grady, C. L., and
Stuss, D. T. (2002). Functional reorganisation of memory after traumatic brain injury: a study with $\mathrm{H}$ 2150 positron emission tomography. J. Neurol. Neurosurg. Psychiatr. 73 173-181.

Levine, B., Fujiwara, E., O’Connor, C. Richard, N., Kovacevic, N., Mandic, M., Restagno, A., Eason, C., Robertson, I. H., Graham, S. J., Cheung, G., Gao, F., Schwartz, M. L., and Black, S. E. (2006). In vivo characterization of traumatic brain injury neuropathology with structural and functional neuroimaging. J. Neurotrauma 23, 1396-1411.

Levine, B., Kovacevic, N., Nica, I., Cheung, G., Schwartz, M. L., and Black, S. E. (2008). Toronto traumatic brain injury study: injury severity and quantified MRI. Neurology 70 , 771-778.

Levine, B., Stuss, D. T., Milberg, W. P., Alexander, M. A., Schwartz, M., and Macdonald, R. (1998). The effects of focal and diffuse brain damage on strategy application: evidence from focal lesions, traumatic brain injury and normal aging. J. Int. Neuropsychol. Soc. 4, 247-264.

MacDonald, A. W. R., Cohen, J. D., Stenger, V. A., and Carter, C. S. (2000). Dissociating the role of the dorsolateral prefrontal and anterior cingulate cortex in cognitive control. Science 288, 1835-1838.

Maruishi, M., Miyatani, M., Nakao, T. and Muranaka, H. (2007). Compensatory cortical activation during performance of an attention task by patients with diffuse axonal injury: a functional magnetic resonance imaging study. J. Neurol. Neurosurg. Psychiatr. 78, 168-173.

Mathias, J. L., and Wheaton, P. (2007). Changes in attention and information-processing speed following severe traumatic brain injury: a meta-analytic review. Neuropsychology 21, 212-223.

Maxwell, W. L., Povlishock, J. T., and Graham, D. L. (1997). A mechanistic analysis of nondisruptive axonal injury: a review. J. Neurotrauma 14, 419-440.

McAllister, T. W., Flashman, L. A., McDonald, B. C., and Saykin, A. J. (2006). Mechanisms of working memory dysfunction after mild and moderate TBI: evidence from functional MRI and neurogenetics. J. Neurotrauma 23, 1450-1467.

McAllister, T. W., Sparling, M. B., Flashman, L. A., Guerin, S. J., Mamourian, A. C., and Saykin, A. J. (2001). Differential working memory load effects after mild traumatic brain injury. Neuroimage 14, 1004-1012.
McIntosh, A. R., Bookstein, F. L., Haxby, J. V., and Grady, C. L. (1996). Spatial pattern analysis of functional brain images using partial least squares. Neuroimage 3, 143-157.

McIntosh, A. R., Chau, W., and Protzner, A. B. (2004). Spatiotemporal analysis of event-related fMRI dada using partial least squares. Neuroimage 23, 764-765.

McIntosh, A. R., and Lobaugh, N. J. (2004). Partial least squares analysis of neuroimaging data: applications and advances. Neuroimage 23(Suppl. 1), S250-S263.

Mesulam, M. M. (1998). From sensation to cognition. Brain 121, 1013-1052.

Mesulam, M. M., Nobre, A. C., Kim, Y.H., Parrish, T. B., and Gitelman, D. R. (2001). Heterogeneity of cingulate contributions to spatial attention. Neuroimage 13, 1065-1072.

Morcom, A. M., Li, J., and Rugg, M. D. (2007). Age effects on the neural correlates of episodic retreival: increased cortical recruitment with matched performance. Cereb. Cortex 17, 2491-2506.

Pandya, D. N., Van Hoesen, G. W., and Mesulam, M. M. (1981). Efferent connections of the cingulate gyrus in the rhesus monkey. Exp. Brain Res. 42, 319-330.

Perlstein, W. M., Cole, M. A., Demery, J. A., Seignourel, P. J., Dixit, N. K., Larson, M. J., and Briggs, R. W. (2004) Parametric manipulation of working memory load in traumatic brain injury: behavioral and neural correlates. J. Int. Neuropsychol. Soc. 10, 724-741.

Petrides, M., and Pandya, D. N. (1999). Dorsolateral prefrontal cortex: comparative cytoarchitectonic analysis in the human and the macaque brain and corticocortical connection patterns. Eur. J. Neurosci. 11, 1011-1036.

Povlishock, J. T. (1992). Traumatically induced axonal injury: pathogenesis and pathobiological implications. Brain Pathol. 2, 1-12.

Povlishock, J. T., and Christman, C. W. (1995). The pathobiology of traumatically induced axonal injury in animals and humans: a review of current thoughts. J. Neurotrauma 12, 555-564.

Sakai, K., and Passingham, R. E. (2006). Prefrontal set activity predicts rulespecific neural processing during subsequent cognitive performance. J. Neurosci. 26, 1211-1218.

Sampson, P. D., Streissguth, A. P., Barr, H. M., and Bookstein, F. L. (1989). Neurobehavioral effects of prenatal alcohol: part II. Partial least squares analysis. Neurotoxicol. Teratol. 11, 477-491. 
Scheibel, R. S., Newsome, M. R., Troyanskaya, M., Steinberg, J. L., Goldstein, F. C., Mao, H., and Levin, H. S. (2009). Effects of severity of traumatic brain injury and brain reserve on cognitive-control related brain activation. J. Neurotrauma 26, 1447-1461.

Scheid, R., Walther, K. R., Guthke, T., Preul, C., and von Cramon, D. Y. (2006). Cognitive sequelae of diffuse axonal injury. Arch. Neurol. 63, 418-424.

Spikman, J. M., Kiers, H. A. L., Deelman, B. G., and van Zomeren, A. H. (2001). Construct validity of concepts of attention in healthy controls and patients with CHI. Brain Cogn. 47, 446-470.

Spikman, J. M., van Zomeren, A. H., and Deelman, B. G. (1996). Deficits of attention after closed head injury: slowness only? J. Clin. Exp. Neuropsychol. 18, 755-767.

Stuss, D. T., and Alexander, M. P. (2007). Is there a dysexecutive syndrome? Philos. Trans. R. Soc. Lond. B Biol. Sci. 362, 901-915.
Stuss, D. T., Binns, M. A., Murphy, K. J., and Alexander, M. P. (2002). Dissociations within the anterior attentional system: effects of task complexity and irrelevant information on reaction time speed and accuracy. Neuropsychology 16, 500-513.

Stuss, D. T., Pogue, J., Buckle, L. and Bondar, J. (1994). Characterization of stability of performance in patients with traumatic brain injury: variability and consistency on reaction time tests. Neuropsychology 8, 316-324.

Stuss, D. T., Stethem, L. L., Hugenholtz, H., Picton, T., Pivik, J., and Richard, M. T. (1989). Reaction time after head injury: fatigue, divided and focused attention, and consistency of performance. J. Neurol. Neurosurg. Psychiatr. 52, 742-748.

Talairach, P., and Tournoux, J. (1988). Co-Planar Stereotaxic Atlas of the Human Brain. Stuttgart: Thieme Medical Publishers.

Teasdale, G., and Jennett, B. (1974). Assessment of coma and impaired consciousness: a practical scale. Lancet 13, 81-84.

Turner, G. R., McIntosh, A. R., and Levine, B. (2011). Prefrontal compensatory engagement in TBI is due to altered functional engagement of existing networks and not functional reorganization. Front. Syst. Neurosci. 5:9. doi:10.3389/fnsys.2011. 00009

Turner, G. T., and Levine, B. (2008). Augmented neural activity during executive control processing following diffuse axonal injury. Neurology 71, 812-818.

Vallesi, A., McIntosh, A. R., and Stuss, D. T. (2011). Over recruitment in the aging brain as a function of task demands: evidence for a compensatory view. J. Cogn. Neurosci. 23, 801-815.

Wager, T. D., and Smith, E. E. (2003). Neuroimaging studies of working memory: a meta-analysis. Cogn. Affect. Behav. Neurosci. 3, 255-274.

Zachary, R. A. (1986). Shipley Institute of Living Scale, Revised Manual.
Los Angeles: Western Psychological Services.

Conflict of Interest Statement: The authors declare that the research was conducted in the absence of any commercial or financial relationships that could be construed as a potential conflict of interest.

Received: 03 February 2011; accepted: 22 November 2011; published online: 09 December 2011.

Citation: Raja Beharelle A, Tisserand $D$, Stuss DT, McIntosh AR and Levine $B$ (2011) Brain activity patterns uniquely supporting visual feature integration after traumatic brain injury. Front. Hum. Neurosci. 5:164. doi: 10.3389/fnhum.2011.00164

Copyright (c) 2011 Raja Beharelle, Tisserand, Stuss, McIntosh and Levine. This is an open-access article distributed under the terms of the Creative Commons Attribution Non Commercial License, which permits non-commercial use, distribution, and reproduction in other forums, provided the original authors and source are credited. 